\title{
Alterstice
}

Revue internationale de la recherche interculturelle

International Journal of Intercultural Research

Revista International de la Investigacion Intercultural

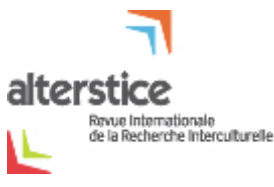

\section{Voeux et mots du bureau de l'ARIC à l'abord de cette nouvelle année}

\section{Bureau de l'Association internationale pour la recherche interculturelle (ARIC)}

Volume 6, numéro 2, 2016

URI : https://id.erudit.org/iderudit/1040627ar

DOI : https://doi.org/10.7202/1040627ar

Aller au sommaire du numéro

Éditeur(s)

Alterstice

ISSN

1923-919X (numérique)

Découvrir la revue

Citer ce document

Bureau de l'Association internationale pour la recherche interculturelle (ARIC)

(2016). Voeux et mots du bureau de l'ARIC à l'abord de cette nouvelle année.

Alterstice, 6(2), 3-5. https://doi.org/10.7202/1040627ar

Droits d'auteur (C) Bureau de l'Association internationale pour la recherche interculturelle (ARIC), 2016
Ce document est protégé par la loi sur le droit d'auteur. L'utilisation des services d'Érudit (y compris la reproduction) est assujettie à sa politique d'utilisation que vous pouvez consulter en ligne.

https://apropos.erudit.org/fr/usagers/politique-dutilisation/ 
RUBRIQU' ARIC

\title{
Voux et mots du bureau de l'ARIC à l'abord de cette nouvelle année
}

\author{
Bureau de l'Association internationale pour la recherche interculturelle (ARIC) ${ }^{1}$
}

L'équipe interculturelle du bureau de I'ARIC veut souligner avec les lectrices et lecteurs d'Alterstice le début de cette année 2017. Tous nos vœux pour qu'elle soit porteuse de relations interculturelles redéployées entre les diverses sociétés et tous les peuples! Depuis quelques années, il apparaît essentiel de retisser des liens plus solides, plus entrelacés et plus diversifiés entre nos cultures, minoritaires et majoritaires, sociales et linguistiques, historiques et politiques... II apparaît aussi que cette responsabilité ne doit pas reposer que sur les systèmes politiques mais que tous les acteurs sociaux ont un rôle à jouer dans ce nouveau "tissage interculturel ». À I'ARIC, et bien modestement, nous offrons des espaces pour que les chercheures et chercheurs, les professionnelles et professionnels, les praticiens et praticiennes, les étudiants et étudiantes et les bénévoles de l'interculturel partagent ensemble leurs savoirs, qu'ils soient disciplinaires, professionnels ou expérienciels, mais aussi pour qu'ils construisent de nouvelles connaissances à partager tant avec les citoyens et citoyennes de leurs sociétés qu'avec les décideurs. Nos publications dans la Collection Espaces interculturels, chez L'Harmattan, ont été nombreuses en 2016 et participent à ces échanges. La revue Alterstice est un autre de ces espaces de diffusion et de construction des savoirs. Nous rappelons à tous les chercheurs du domaine interculturel qu'ils peuvent proposer des textes pour la revue, pour enrichir notre patrimoine de recherche interculturelle commun. Si le colloque de Recife a représenté un moment fort pour l'ARIC en 2016, nous attendons avec impatience le congrès ARIC 2017 qui se tiendra à Antananarivo à Madagascar du 23 au 27 mai 2017. Que vous ayez ou non fait une proposition de communication, vous êtes toutes et tous invités à y participer, là encore dans l'esprit de co-construction des savoirs qui représente la spécificité de notre association. Sur le thème "Nouveaux contextes et nouvelles pratiques interculturelles. Globalisation et circulation ", le congrès organisé par le Centre d'études et de recherche sur les constructions identitaires de I'Université d'Antananarivo offrira des moments d'échange et d'apprentissage sur la circulation des idées, des ressources et des biens mais aussi sur la mobilité humaine, les déplacements de population, les redéfinitions contemporaines des frontières politiques, économiques et linguistiques, et finalement sur les nouveaux rapports à l'altérité qui se dessinent au travers des discours sociaux, médiatiques ou politiques en lien avec la l'insécurité, les peurs, les identités etc. Plusieurs moments importants sont prévus en plus des activités scientifiques durant ce congrès, dont l'assemblée générale de l'ARIC, la remise du prix ARIC 2017 de la meilleure thèse en recherche interculturelle, une journée de visites culturelles et aussi... un forum public "Femmes et féminismes à Madagascar : vers un dialogue interculturel... ». Venez nombreuses et nombreux à cette nouvelle occasion d'apprendre, de développer vos contacts et de faire entendre votre voix dans les réseaux internationaux de la recherche interculturelle! Alors pour cette année 2017, je nous souhaite un super-congrès qui fasse avancer la recherche interculturelle mais aussi son application dans des pratiques et politiques nationales et internationales qui ont tout à y gagner! Et puis, dans la suite, de nombreuses publications, des collaborations, des subventions internationales... et encore plus de recherche interculturelle !

Michèle Vatz Laaroussi, présidente de I'ARIC

Chères lectrices et chers lecteurs d'Alterstice, la trésorière et le secrétaire de I'ARIC vous souhaitent depuis Fribourg une excellente année 2017. Nous vous souhaitons une année " propre en ordre ", pour reprendre une expression bien suisse. Qu'elle soit propre aux rencontres, personnelles, professionnelles et scientifiques, et aux échanges, particulièrement interculturels, interdisciplinaires, internationaux. Propre à développer les relations entre chercheurs et professionnels œuvrant dans le champ de l'interculturel, au-delà des situations et réalités particulières à chacun et chacune. Qu'elle soit en ordre, mais en ordre dispersé afin de développer 
chacun et chacune dans nos contextes un ensemble de projets couvrant des horizons variés, ayant pour trait commun une volonté de construire des ponts entre cultures et appartenances diverses. En ordre de bataille aussi, afin de lutter par nos actions et engagements contre les tendances au repli identitaire et à la peur de l'autre, afin d'œuvrer inlassablement par nos activités professionnelles et scientifiques au partage de connaissances et de savoirs. Ces vœux sont aussi l'occasion pour nous de remercier chaleureusement les membres de I'ARIC pour leur participation et investissement à la vie de notre association et nous invitons tous ceux et celles qui ont croisé notre route à un moment donné à rejoindre l'association (http://www.unifr.ch/ipg/aric/). Pour nourrir votre réflexion à ce sujet, nous vous rappelons que l'ARIC a pour mission de promouvoir la collaboration interdisciplinaire et interculturelle, de faciliter l'échange d'informations entre ses membres et d'établir, dans l'espace francophone, un réseau international et interdisciplinaire. L'association a également pour objectif d'encourager la recherche interculturelle, de faciliter l'articulation entre théorie et pratique interculturelle, de promouvoir et diffuser des publications et revues scientifiques dans les thématiques de l'interculturel et d'organiser des rencontres scientifiques d'envergure nationale internationale. À ce jour, I'ARIC compte environ 150 membres - chercheures et chercheurs, praticiennes et praticiens, étudiantes et étudiants - provenant majoritairement de France, du Canada, d'Algérie et de Suisse, mais aussi de Belgique, du Brésil, du Maroc, de Tunisie, d'Argentine, d'Inde, d'Allemagne, d'Italie, de République démocratique du Congo, du Cambodge, du Portugal, de Roumanie, de République tchèque, de Norvège ou d'Espagne. En 2017, nous vous invitons à participer au $16^{\mathrm{e}}$ congrès international de l'ARIC à Madagascar. Tous nos meilleurs vœux pour l'année 2017 : que cette année vous soit riche et fructueuse !

Alida Gulfi, trésorière, et Xavier Conus, secrétaire administratif.

Je joins mes vœux à ceux des autres membres du bureau de l'ARIC pour souhaiter aux lectrices et lecteurs d'Alterstice une excellente année 2017. Cette année 2017 sera d'ailleurs l'occasion pour l'ARIC de chercher à développer sa communication à la fois entre ses membres et vers l'extérieur. Garantir la diffusion et la possibilité de partager nos productions, événements, activités et recherches à l'ère des télécommunications et de la numérisation devient d'une importance vitale si nous voulons nous positionner comme une association reconnue sur la scène internationale. C'est pourquoi le site internet de l'ARIC fera peau neuve en 2017. Nous travaillons actuellement à la construction d'un tout nouveau site qui offrira diverses possibilités aux utilisateurs, dont un intranet qui permettra le partage exclusif de documents, images ou vidéos aux membres de I'ARIC. Réalisé a un prix très concurrentiel par une conceptrice graphique qui est d'ailleurs aussi médiatrice interculturelle, ce qui l'aide à bien comprendre les besoins de notre association dédiée aux thèmes et enjeux de l'interculturel, notre nouvelle page internet sera en fonction bien avant le prochain congrès de l'association en mai à Madagascar. Ce nouvel outil de communication permettra de développer également d'autres opportunités d'échange entre les membres de I'ARIC et les autres chercheurs, décideurs politiques, partenaires, mais aussi avec le grand public, par exemple par le biais d'un calendrier des événements susceptibles d'intéresser notre communauté, ou par l'information au sujet des parutions d'ouvrages publiés par nos membres. Un lien entre le site internet et la page Facebook de l'association facilitera l'accès aux utilisateurs des réseaux sociaux, et garantira en même temps une large visibilité à l'information ainsi partagée avec le monde. La commission Communications de l'ARIC est fière d'avoir conclu un projet longuement réfléchi par les divers membres s'étant succédé au Conseil de notre association, qui continue ainsi de se montrer créative et avant-gardiste.

Linamar Campos Flores, secrétaire générale

En m'associant aux vœux de l'ensemble du bureau, je termine cette Rubriqu'ARIC par quelques informations concernant des publications en lien avec l'association. Dans la suite des travaux présentés au Colloque ARIC 2016, cinq ouvrages viendront compléter la grande liste de productions de I'ARIC. Tout d'abord, l'ouvrage bilingue français/portugais $O$ Psicólogo frente ao desafio tecnológico, novas identidades, novos campos, novas práticas (ISBN : 978-85-415-0752-3) sous la direction d'Elaine Costa Fernandez et Véronique Donard, réunit des textes originaux sur l'usage fait par des psychologues des Nouvelles Technologies de l'Information et de la Communication (NTIC). Outre la belle préface de Patrick Denoux, le lecteur trouvera trois parties consacrées aux politiques publiques à la recherche et la pratique professionnelle du psychologue dans la contemporanéité. Parallèlement le Cahier de résumés (ISBN : 978-85-415-0744-8) distribué aux participants à Olinda contient plus de 300 résumés autour des thématiques de la mobilité, des réseaux et de l'interculturalité. II reste une 
référence précieuse pour les doctorants et futurs chercheurs. Ensuite, deux ouvrages avec des travaux complets en portugais seront publiés début 2017 aux Éditions universitaires de l'Université Fédérale de Pernambuco (UFPE/Recife). Le premier, sous la direction de Candy Marques Laurendon, Elaine CostaFernandez et Rosiane Xypas avec préface de Reinaldo Fleury et postface de Lucienne Martins Borges, abordera les questions liées à l'espace et au territoire, la mobilité humaine, les politiques publiques et les réseaux de soin, réaffirmant la dimension pluridisciplinaire de l'ARIC. L'autre ouvrage, sous la direction de Rosiane Xypas, Elaine Costa-Fernandez et Candy Marques Laurendon, visera davantage les questions posées par l'interculturalité et la communication, que ce soit en contexte scolaire, dans la formation professionnelle, la création artistique ou les médias. La préface sera rédigée par Rosalina Maria Sales Chianca, présidente de l'Association brésilienne des professeurs de français, et la postface par Constantin Xypas, spécialiste international de l'épistémologie de Jean Piaget. Finalement un ouvrage en français sous la coordination de Elaine Costa Fernandez, Patrick Denoux et Odette Lescarret sera publié dans la collection Espaces interculturels à L'Harmattan courant 2017. Outre la préface de Benoit Virole et la postface de Julien Teyssier, il réunira des textes spécialement choisis pour la pertinence et l'originalité de leurs approches dans le domaine de la recherche interculturelle.

Elaine Costa Fernandez, vice-présidente

\section{Rattachement des auteurs}

Les auteurs constituent le bureau (l'exécutif) de l'Association internationale pour la recherche interculturelle.

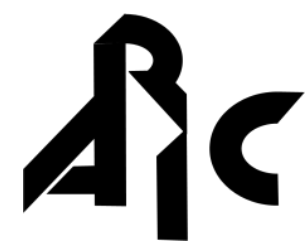

\section{Correspondance}

aric_assoc@yahoo.fr

\section{Pour citer cet article}

Bureau de l'ARIC. (2016). Vœux et mots du bureau de l'ARIC à l'abord de cette nouvelle année [Rubriqu'ARIC]. Alterstice, 6(2), 3-6. 